\title{
Association of ZNF644, GRM6, and CTNND2 genes with high myopia in the Han Chinese population: Jiangsu Eye Study
}

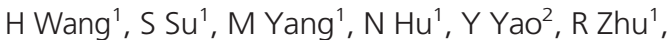
J Zhou' ${ }^{1}$, C Liang ${ }^{3}$ and H Guan ${ }^{1}$

\section{Abstract}

Aims High myopia is a common visual disorder in the world. The ZNF644, GRM6, and CTNND2 genes are expressed in the retina. This study aims to investigate the associations of these genes with high myopia in Han Chinese population. Methods The case-control association included high myopia cases $(n=430)$ and controls $(n=430)$ recruited from a populationbased study, 'Jiangsu Eye Study'. Fourteen single-nucleotide polymorphisms (SNPs) in three genes were genotyped by the TaqMan method using the real-time PCR system. Results Three SNPs GRM6-rs11746675, GRM6-rs2067011, and GRM6-rs2645339 were associated with high myopia (odds ratio $(\mathrm{OR})=0.74, P=0.003 ; \mathrm{OR}=0.78, P=0.018$; and $\mathrm{OR}=0.78, P=0.023$; respectively). The significances of rs2067011 and rs2645339 disappeared after multiple testing corrections. Rs11746675 remained significant after correction for multiple testing. The genetic model analysis found that GRM6-rs11746675 and GRM6-rs2067011 were suggestively associated with high myopia in the recessive model $(\mathrm{OR}=0.54$, $P=0.004 ; \mathrm{OR}=0.52, P=0.003$; respectively). Haplotype GAT for GRM6 markers rs2067011-rs2645339-rs762724 showed significance $(P=0.0239)$, but such association did not remain significant after multiple testing corrections. Conclusions Our data suggested that genetic variants in GRM6 are associated with high myopia. The mechanism of GRM6 in the development of high myopia need to be further investigated.

Eye (2016) 30, 1017-1022; doi:10.1038/eye.2016.8; published online 1 April 2016

\section{Introduction}

Myopia is a common visual disorder in the worldwide with significant concerns in public health, especially in East Asian populations. High myopia ( -6.00 diopters (D) or less) is one of the leading cause of vision loss or even irreversible blindness with pathologic complications such as myopic retinopathy, maculopathy, choroidal neovascularization, retinal detachment, posterior staphyloma, cataract, and primary open-angle glaucoma. ${ }^{1}$

The prevalence of myopia varies across ethnic groups and has rapidly increased in the past few decades. Among adult Chinese in the United States, the prevalence of myopia (spherical equivalent $(\mathrm{SE}) \leq-1.0 \mathrm{D})$ and high myopia (SE $\leq-5.0 \mathrm{D})$ was 37.2 and $11.8 \%$, respectively. ${ }^{2}$ In Singapore adults, the prevalence of myopia ( $\mathrm{SE} \leq-0.5 \mathrm{D})$ and high myopia $(\mathrm{SE} \leq-5.0 \mathrm{D})$ was 38.9 and $8.4 \%$, respectively. ${ }^{3}$ Chinese adults had higher prevalence of myopia and high myopia compared with non-Chinese adults in above surveys. A study reported that the prevalence of myopia ( $\mathrm{SE}<-0.5 \mathrm{D})$ and high myopia $(\mathrm{SE}<-6.0 \mathrm{D})$ was 95.5 and $19.5 \%$, respectively, in college students of Shanghai. ${ }^{4}$ In the long term, such problems will impose a heavy burden on the health-care system and the economy of the society concerned.

Myopia is a multifactorial disease. ${ }^{1}$ Familial aggregation studies have estimated sibling recurrence risks of common forms of refractive errors to range from 3 to 5 for myopia. ${ }^{5}$ Recent twin study of myopia estimated heritabilty was $78 \%$ for SE in Koreans. ${ }^{6}$ So far, almost 22 loci (MYP1-MYP3, MYP5-MYP23) have been identified for myopia susceptibility through family-based linkage analyses and genome-wide association studies. Unfortunately, no candidate gene has been consistently replicated.
${ }^{1}$ Eye Institute, Affiliated Hospital of Nantong University, Nantong, China

${ }^{2}$ Affiliated Wuxi People's Hospital of Nanjing Medical University, Wuxi, China

${ }^{3}$ Funing County Center for Disease Prevention and Control, Yancheng, China

Correspondence:

H Guan, Eye Institute, Affiliated Hospital of Nantong University, 20 Xisi Road, Nantong 226001, China

Tel: +86 1380908 8972; Fax: +86051385519820. E-mail: guanhjeye@163. com

Received: 24 January 2015 Accepted in revised form: 3 December 2015 Published online: 1 April 2016 
Although the pathophysiology of high myopia is far from clearly understood, a common pathologic structural abnormality is the excessive lengthening of the posterior segment of the ocular globe. A visually triggered signaling cascade from the retina ultimately guides the scleral remodeling that leads to eye growth. ${ }^{7}$ The zinc-finger protein 644 gene (ZNF644) is a zinc-finger protein that functions as a transcriptional factor and is expressed in the retina. ${ }^{8}$ The glutamate receptor metabotropic 6 gene (GRM6) is important in the function of the ON-bipolar cells, which is a major controller of dopamine release. ${ }^{9}$ The catenin (cadherin-associated protein), delta 2 (CTNND2) encodes an adhesive junctionassociated protein of the armadillo/beta-catenin superfamily and is implicated in brain and eye development and cancer formation. ${ }^{10}$ Here we selected 14 common single-nucleotide polymorphisms (SNPs) of ZNF644, GRM6, and CTNND2 to evaluate whether these gene variations are associated with high myopia in Han Chinese populations.

\section{Materials and methods}

The project was approved by the ethics committee of Affiliated Hospital of Nantong University. A written informed consent was obtained from each participant. The research followed the tenets of the Declaration of Helsinki.

This study was a part of the Jiangsu Eye Study, a population-based epidemiologic study focusing on common eye diseases and health-related parameters. ${ }^{11}$ One rural district/county within southern and northern Jiangsu was selected as the sampling area, which are Binhu District and Funing County, respectively. The surveys were carried out by randomly selecting individuals within each district/county which was similar with the method we described previously. ${ }^{12}$ Sample size was based on estimating an anticipated $2.6 \%$ prevalence for $\mathrm{SE} \leq-6.0 \mathrm{D}$ within an error bound (precision) of $25 \%$ with 95\% confidence interval (CI). ${ }^{13}$ Assuming an examination response rate of $85 \%$, and a design effect of 1.5 to account for inefficiencies associated with the cluster sampling design, a sample of 4062 persons was required for each district/county. The participants were unrelated and self-identified Han Chinese. All individuals were randomly selected from each district/county. Geographically defined cluster sampling included 6722 individuals in Binhu District from January to December 2010. Actually, 6106 subjects were examined with the response rate of $90.8 \%$. The same sampling was used in 6145 randomly selected individuals in Funing County from September 2010 to May 2011 and actually 5947 subjects were examined with the response rate of $96.8 \%$.

All study participants underwent a detailed ocular examination. The participants underwent a noncycloplegic refraction test with auto refractometer
(AR-610; NIDEK, Aichi, Japan), subjective refraction and retinoscope. The inclusion criteria for high myopia included: (i) myopia in both eyes and a SE $\leq-6.00 \mathrm{D}$ in at least one eye, ${ }^{14}$ (ii) without nuclear cataract, (iii) without corneal lesions, (iv) without any previous history of ocular procedures. Finally, we examined 430 high myopia patients. The criteria of control individuals in this study had uncorrected visual acuity of 20/20 or better by Early Treatment Diabetic Retinopathy Study (ETDRS) chart and without other known eye or systemic diseases. In all, 430 individuals were included as normal controls. The ocular examinations were performed by professionals according to the uniform standard. Peripheral venous blood was collected in an ethylene diamine tetraacetic acid anti-coagulation tube. Genomic DNA was isolated from leukocytes by the phenol-chloroform method. Details of ocular examination, blood collection, and DNA extraction have been reported previously. ${ }^{11}$

\section{Selection and genotyping of SNPs}

Fourteen SNPs were selected from three candidate genes (ZNF644, GRM6, CTNND2). We selected haplotype-tagging SNP by searching Han Chinese data from the International HapMap Project (http:/ / hapmap. ncbi.nlm.nih.gov/) using the Tagger program. All SNPs were selected with a minor-allele frequency $>5 \%$ in the HapMap CHB population. Details of tested SNPs and TaqMan assays are listed in Table 1. Genotyping of all SNPs were conducted with a commercial gene-expression assay (TaqMan Assay; Applied Biosystems, Foster City, CA, USA, with the 7500 Real-Time PCR System; Applied Biosystems), as described in our previous publication. ${ }^{11}$

\section{Statistical analysis}

Statistical analyses were performed with a commercial statistical software program (Stata 8.0; Stata Corp, College

Table 1 Taqman SNP genotyping assays information

\begin{tabular}{llllll}
\hline Gene & SNP ID & Chromosome & $\begin{array}{l}\text { Minor/ } \\
\text { major }\end{array}$ & Maf & SNP type \\
\hline ZNF644 & rs358693 & Chr1 & A/C & 0.385 & Intron \\
ZNF644 & rs358695 & Chr1 & A/G & 0.263 & Intron \\
ZMF644 & rs358698 & Chr1 & T/C & 0.384 & Intron \\
ZNF644 & rs358690 & Chr1 & A/G & 0.302 & Intron \\
ZNF644 & rs12724992 & Chr1 & G/A & 0.143 & Intron \\
GRM6 & rs11746675 & Chr5 & T/C & 0.426 & Silent mutation \\
GRM6 & rs17078853 & Chr5 & G/T & 0.057 & UTR 3' \\
GRM6 & rs762724 & Chr5 & C/T & 0.463 & Intron \\
GRM6 & rs2067011 & Chr5 & A/G & 0.472 & Missense mutation \\
GRM6 & rs2645339 & Chr5 & G/A & 0.476 & Nonsense mutation \\
GRM6 & rs2071247 & Chr5 & T/C & 0.253 & Silent mutation \\
CTNND2 & rs1479617 & Chr5 & A/G & 0.407 & Intron \\
CTNND2 & rs6885224 & Chr5 & C/T & 0.348 & Intron \\
CTNND2 & rs12716080 & Chr5 & G/T & 0.468 & Intron \\
\hline
\end{tabular}


Station, TX, USA). $\chi^{2}$ was used to test the allelic and genotypic associations between cases and controls and to estimate odds ratio (OR) and 95\% CI. Hardy-Weinberg equilibrium (HWE) of each SNP in control subjects were also tested using a $\chi^{2}$ test. Correction for multiple comparisons was performed by permutation. Multiple testing was corrected by generating empirical $P$-values based on 50000 permutations across all SNPs and haplotype blocks for a given sample set as appropriate. The haplotype blocks were estimated by using software Haploview Version 4.2 (Mark Daly's Laboratory, Cambridge, MA, USA). $P<0.05$ was considered as statistically significant.

\section{Results}

The demographic information for the study participants was generalized in Table 2. All of the tested SNPs are in HWE in the control population, except GRM6-rs17078853 that was excluded from further analysis. The call rates of all SNPs genotyping were $>98 \%$.

In allele frequency analysis (Table 3 ), three SNPs of GRM6 were associated with high myopia $(P<0.05)$ : rs11746675 (OR $=0.74, P=0.003,95 \% \mathrm{CI}: 0.60-0.91)$, rs2067011 (OR $=0.78, P=0.018,95 \% \mathrm{CI}: 0.63-0.96)$, rs2645339 (OR $=0.78, P=0.023,95 \% \mathrm{CI}: 0.63-0.96)$. The frequency of the minor $\mathrm{T}$ allele of rs11746675 was
$35.9 \%$ in the high myopia group and $42.9 \%$ in the control group, indicating a protective role. In addition, this SNP of GRM6 remained significant $\left(P_{\mathrm{C}}=0.009\right)$, whereas the significance of other two SNPs disappeared after correction for permutation test.

In genotype-frequency analysis (Table 4), GRM6rs11746675, GRM6-rs762724, GRM6-rs2067011, and GRM6-rs2645339 had significant differences between cases and controls in recessive model $(P=0.004, P=0.03$, $P=0.003, P=0.005$, respectively). The frequency of the TT genotype of rs11746675 was $10.9 \%$ in the high myopia group and $18.1 \%$ in the control group and conferred an OR of 0.54 in the recessive model, indicating a protective role in developing high myopia.

In haplotype analysis (Table 5), four SNPs of ZNF644, three SNPs of GRM6 genotyped in this study were in the same LD block. One haplotype block of GRM6 showed statistical significance between cases and controls: GAT (SNP rs2067011-rs2645339-rs762724, $P=0.024$ ). However, their significance disappeared after correction for multiple testing $\left(P_{\mathrm{C}}=0.08\right)$.

\section{Discussion}

This study presented the evidence of involvement of GRM6 in the susceptibility of high myopia. Our subjects

Table 2 Demographic information of study participants

\begin{tabular}{|c|c|c|c|c|c|c|}
\hline \multirow[t]{2}{*}{ Group } & \multirow[t]{2}{*}{$n$} & \multirow[t]{2}{*}{ Age (mean $\pm S D$; years) } & \multicolumn{2}{|c|}{ Gender } & \multicolumn{2}{|c|}{ Spherical equivalent } \\
\hline & & & Female (\%) & Male (\%) & $O D$ & OS \\
\hline Control & 430 & $59.37 \pm 4.06$ & $265(61.63)$ & $165(38.37)$ & NA & NA \\
\hline High myopia & 430 & $65.72 \pm 7.49$ & $277(64.42)$ & $153(35.58)$ & $-10.73 \pm 3.32$ & $-10.52 \pm 3.24$ \\
\hline
\end{tabular}

Table 3 Distribution of minor allele of tested 14 SNPs and their association with high myopia

\begin{tabular}{|c|c|c|c|c|c|}
\hline Gene name & SNPs minor/major & Controls minor/major (\%) & Cases minor/major (\%) & OR $(95 \% C I)$ & P-value \\
\hline \multirow[t]{5}{*}{ ZNF644 } & rs358693 A/C & $320 / 540(37.2)$ & 290/570 (33.7) & $0.94(0.76-1.17)$ & 0.13 \\
\hline & rs358695 A/G & $372 / 488(43.3)$ & $401 / 459(46.6)$ & $1.12(0.91-1.37)$ & 0.16 \\
\hline & rs358698 T/C & $324 / 536(37.7)$ & $291 / 569(33.8)$ & $0.92(0.74-1.14)$ & 0.10 \\
\hline & rs358690 A/G & 134/726 (15.6) & $114 / 746(13.3)$ & $0.84(0.63-1.13)$ & 0.17 \\
\hline & rs12724992 G/A & 47/813 (5.5) & $57 / 803(6.6)$ & $1.36(0.88-2.10)$ & 0.31 \\
\hline \multirow[t]{6}{*}{ GRM6 } & rs11746675 T/C & $369 / 491(42.9)$ & $309 / 551(35.9)$ & $0.74(0.60-0.91)$ & $0.003 / 0.005(\mathrm{~Pa})$ \\
\hline & rs17078853 G/T & $110 / 750(12.8)$ & $117 / 743$ (13.6) & - & - \\
\hline & rs762724 C/T & $351 / 509(40.8)$ & $311 / 549(36.2)$ & $0.82(0.66-1.01)$ & 0.048 \\
\hline & rs2067011 A/G & $357 / 503(41.5)$ & $309 / 551$ (35.9) & $0.78(0.63-0.96)$ & $0.018 / 0.021(\mathrm{~Pa})$ \\
\hline & rs2645339 G/A & $359 / 501(41.7)$ & $313 / 547(36.4)$ & $0.78(0.63-0.96)$ & $0.023 / 0.021(\mathrm{~Pa})$ \\
\hline & rs2071247 T/C & $325 / 535(37.8)$ & $334 / 526(38.8)$ & $1.04(0.84-1.29)$ & 0.66 \\
\hline \multirow[t]{3}{*}{ CTNND2 } & rs1479617 A/G & $224 / 636(26.0)$ & $213 / 647(24.8)$ & $0.95(0.75-1.2)$ & 0.54 \\
\hline & rs6885224 C/T & $180 / 680(20.9)$ & 192/668 (22.3) & $1.04(0.81-1.34)$ & 0.48 \\
\hline & rs12716080 G/T & 183/677 (21.3) & 205/655 (23.8) & $1.11(0.87-1.43)$ & 0.20 \\
\hline
\end{tabular}

Abbreviation: Pa, after age correction. All HWE $P>0.05$ except GRM6-rs17078853 $P<0.05$. 
Table 4 Genotype frequency of SNPs in control and high myopia subjects

\begin{tabular}{|c|c|c|c|c|c|c|c|}
\hline \multirow{2}{*}{ SNP } & \multicolumn{3}{|c|}{ Genotype distribution $n(\%)$} & \multicolumn{2}{|c|}{ Dominant model } & \multicolumn{2}{|c|}{ Recessive model } \\
\hline & & Control & High myopia & OR $(95 \% C I)$ & $\mathrm{Pa}$ & OR $(95 \% C I)$ & $\mathrm{Pa}$ \\
\hline \multirow[t]{3}{*}{ GRM6-rs11746675 } & $\mathrm{CC}$ & $139(32.3)$ & $168(39.1)$ & \multirow[t]{3}{*}{$0.74(0.55-1.00)$} & \multirow[t]{3}{*}{0.05} & \multirow[t]{3}{*}{$0.54(0.35-0.83)$} & \multirow[t]{3}{*}{0.004} \\
\hline & TC & $213(49.5)$ & $215(50.0)$ & & & & \\
\hline & TT & $78(18.1)$ & $47(10.9)$ & & & & \\
\hline \multirow[t]{3}{*}{ GRM6-rs762724 } & TT & $152(35.3)$ & $166(38.6)$ & \multirow[t]{3}{*}{$0.85(0.63-1.15)$} & \multirow[t]{3}{*}{0.29} & \multirow[t]{3}{*}{$0.62(0.40-0.95)$} & \multirow[t]{3}{*}{0.03} \\
\hline & TC & $205(47.7)$ & $217(50.5)$ & & & & \\
\hline & $\mathrm{CC}$ & $73(17.0)$ & $47(10.9)$ & & & & \\
\hline \multirow[t]{3}{*}{ GRM6-rs2067011 } & GG & $149(34.7)$ & $166(38.6)$ & \multirow[t]{3}{*}{$0.83(0.61-1.12)$} & \multirow[t]{3}{*}{0.23} & \multirow[t]{3}{*}{$0.52(0.34-0.81)$} & \multirow[t]{3}{*}{0.003} \\
\hline & GA & $205(47.7)$ & $219(50.9)$ & & & & \\
\hline & $\mathrm{AA}$ & $76(17.7)$ & $45(10.5)$ & & & & \\
\hline \multirow[t]{3}{*}{ GRM6-rs2645339 } & $\mathrm{AA}$ & $147(34.2)$ & $165(38.4)$ & \multirow[t]{3}{*}{$0.82(0.61-1.11)$} & \multirow[t]{3}{*}{0.20} & \multirow[t]{3}{*}{$0.54(0.35-0.84)$} & \multirow[t]{3}{*}{0.005} \\
\hline & GA & $207(48.1)$ & $217(50.5)$ & & & & \\
\hline & GG & 76 (17.7) & 48 (11.2) & & & & \\
\hline
\end{tabular}

Abbreviation: Pa, after age correction.

Table 5 Haplotype analysis of ZNF644 and GRM6 in control and high myopia subjects

\begin{tabular}{|c|c|c|c|c|c|}
\hline \multirow{2}{*}{ Gene name } & \multirow{2}{*}{ Haplotype } & \multicolumn{2}{|c|}{ Haplotype frequency } & \multirow{2}{*}{ OR $(95 \% C I)$} & \multirow{2}{*}{ P-value } \\
\hline & & Controls & Cases & & \\
\hline \multirow[t]{4}{*}{ ZNF644 (rs358695-rs358693-rs358698-rs358690) } & ACCG & 0.43 & 0.46 & $1.15(0.95-1.39)$ & 0.16 \\
\hline & GATG & 0.22 & 0.21 & $0.90(0.71-1.12)$ & 0.36 \\
\hline & GCCG & 0.19 & 0.20 & $1.04(0.81-1.32)$ & 0.77 \\
\hline & GATA & 0.15 & 0.13 & $0.86(0.66-1.13)$ & 0.29 \\
\hline \multirow[t]{2}{*}{ GRM6 (rs2067011-rs2645339-rs762724) } & GAT & 0.58 & 0.63 & $1.25(1.03-1.52)$ & $0.024 / 0.08(\mathrm{Pc})$ \\
\hline & AGC & 0.40 & 0.36 & $0.83(0.68-1.00)$ & 0.05 \\
\hline
\end{tabular}

Abbreviation: Pc, after permutation correction.

were from the Jiangsu Eye Study, a population-based epidemiologic study. The design of a population-based study can minimize the sample-selection bias often present in a hospital-based case-control study.

Previous studies indicate that genes responsive to visual signals are involved in the biological pathways of ocular growth. ${ }^{7}$ The abnormality of sclera in highly myopic eyes of human and animal models is a result of changes in scleral extracellular matrix metabolism. ${ }^{15}$ Therefore, we hypothesize that genes directly responsive to visual signals such as ZNF644, GRM6 and CTNND2 might functionally contribute to the development of high myopia. ${ }^{8-10}$

GRM6 mapped to 5q35, contained 10 exons and encoded an 877 amino acid protein, mGluR6. As a member of Group III mGluRs (mGluR4, 6, 7, and 8), it contains a signal peptide, a large bi-lobed extracellular $\mathrm{NH} 2$-terminal domain containing the glutamate-binding site, seven $G$ protein-coupled receptor transmembrane domains and an intracellular $\mathrm{COOH}$-terminal domain. Mutations in GRM6 have been identified in patients with congenital stationary night blindness (CSNB) in previous studies. ${ }^{9}$ High myopia is usually observed in
CSNB1B patients with GRM6 mutations, ${ }^{16}$ and myopia is not always associated with CSNB, except in cases resulting from mutations in NYX and GRM6. ${ }^{17,18}$ $\mathrm{Xu}$ et $a l^{16}$ identified three novel variations with potential functional consequences in the GRM6 of patients with high myopia, suggesting a potential role in the development of myopia in rare cases. Our findings support GRM6 as a susceptibility gene for high myopia.

An essential step in intricate visual processing is the segregation of visual signals into $\mathrm{ON}$ and OFF pathways by retinal bipolar cells. ${ }^{19}$ A mouse model lacking the GRM6 gene showed a loss of ON response, but an unchanged OFF response to light, demonstrating its essential role in ON synaptic transmission. ${ }^{20}$ Disruption of $\mathrm{ON}$ and $\mathrm{OFF}$ pathway transmission perhaps alter eye growth. GRM6 is important in the function of the ON-bipolar cells, which is a major controller of dopamine release. This may be important because the evidence on experimental myopia suggests that dopamine release may be an important factor in the control of eye growth. However, the specific mechanism is not clear and need to be investigated in the future. 
ZNF644 belongs to the Krüppel C2H2-type zinc-finger protein family, which contains $7 \mathrm{C} 2 \mathrm{H} 2$-type zinc fingers. Shi et $a l^{8}$ first used exome sequencing to identify mutations in zinc-finger protein 644 in a large pedigree with autosomal dominant high myopia, and then replicated in a Chinese cohort. ZNF644 is predicted to be a transcription factor and is expressed in the retina. It may regulate genes involved in eye development, a mutant ZNF644 protein may impact the normal eye development and therefore underlie the axial elongation of the eye globe in high myopia patients. 8,21 Tran-Viet et $a l^{21}$ identified two novel variants in ZNF644 in US cohort, in addition to a known variant that demonstrated association. Mutations of ZNF644 identified in Mendelian inheritance families and sporadic cases, explain only a small proportion of the subjects with high myopia. Therefore, we evaluated in a larger cohort of patients with well-characterized high myopia and normal controls to determine whether these variants are associated with the clinical outcome or not. However, we did not find the association of the selected SNPs of ZNF644 with high myopia in this study.

CTNND2 gene on chromosome 5p15 previously found to be linked to high myopia in a family segregation study of three Hong Kong Chinese pedigrees (LOD =4.68). ${ }^{22}$ This gene encodes an adhesive junction-associated protein of the armadillo/beta-catenin superfamily and is implicated in brain and eye development and cancer formation. ${ }^{10}$ Two SNPs (rs12716080 and rs6885224) of CTNND2 in the metaanalysis of Singaporean Chinese data sets were identified strong association with high myopia. ${ }^{23}$ However, Yu et al ${ }^{24}$ failed to replicate the association in a Chinese Population. Instead, they suggested that mutation in the rs1479617 region of CTNND2 may be important for pathological myopia development. ${ }^{24}$ In our study, we failed in the repetition of these three SNPs (rs12716080, rs6885224, and rs11479617) in the role of myopia.

The possible explanations to the inconsistency of the association studies could be the differences in the sample sizes of subjects, the recruitment criteria, and/or ethnic groups recruited in the studies. Our study has certain limitations. With older age, several age-related confounding factors may come to play. But we make strict standard to select the group. It also can avoid the influence of some environmental factors, for example, outdoor activities, near work, education. Because most of the subjects are farmers.

In conclusion, we found that GRM6 genetic variants are associated with the risk of high myopia in Chinese population. To further confirm the role of GRM6 in the pathogenesis of high myopia, the current results from this population-based study will serve as the baseline for prospective observation of the role of genetic factors in the development of high myopia.

\section{Summary}

What was known before

- High myopia is a common visual disorder in the world. The ZNF644, GRM6, and CTNND2 genes are expressed in the retina.

What this study adds

- We have investigated the associations of ZNF644, GRM6, and CTNND2 genes with high myopia and concluded that GRM6 gene might be involved in high myopia pathogenesis in the Han Chinese population.

\section{Conflict of interest}

The authors declare no conflict of interest.

\section{Acknowledgements}

We are grateful to all the subjects for participating in our myopia genetics study. We appreciate the great contribution of Affiliated Wuxi People's Hospital of Nanjing Medical University, Funing Health Bureau, Funing County Center for Disease Prevention and Control, Shizhuang Eye Hospital of Funing and the People's Hospital of Funing in study coordination and participant recruitment. This study was funded by Nantong University.

\section{References}

1 Morgan IG, Ohno-Matsui K, Saw SM. Myopia. Lancet 2012; 379(9827): 1739-1748.

2 Pan CW, Klein BE, Cotch MF, Shrager S, Klein R, Folsom A et al. Racial variations in the prevalence of refractive errors in the United States: the multi-ethnic study of atherosclerosis. Am J Ophthalmol 2013; 155(6): 1129-1138.

3 Pan CW, Zheng YF, Anuar AR, Chew M, Gazzard G, Aung T et al. Prevalence of refractive errors in a multiethnic Asian population: the Singapore epidemiology of eye disease study. Invest Ophthalmol Vis Sci 2013; 54(4): 2590-2598.

4 Sun J, Zhou J, Zhao P, Lian J, Zhu H, Zhou Y et al. High prevalence of myopia and high myopia in 5060 Chinese university students in Shanghai. Invest Ophthalmol Vis Sci 2012; 53(12): 7504-7509.

5 Fotouhi A, Etemadi A, Hashemi H, Zeraati H, Bailey-Wilson JE, Mohammad $\mathrm{K}$ et al. Familial aggregation of myopia in the Tehran eye study: estimation of the sibling and parent offspring recurrence risk ratios. Br J Ophthalmol 2007; 91(11): 1440-1444.

6 Kim MH, Zhao D, Kim W, Lim DH, Song YM, Guallar E et al. Heritability of myopia and ocular biometrics in Koreans: the healthy twin study. Invest Ophthalmol Vis Sci 2013; 54(5): 3644-3649.

7 Jacobi FK, Zrenner E, Broghammer M, Pusch CM. A genetic perspective on myopia. Cell Mol Life Sci 2005; 62(7-8): 800-808.

8 Shi Y, Li Y, Zhang D, Zhang H, Li Y, Lu F et al. Exome sequencing identifies ZNF644 mutations in high myopia. PLoS Genet 2011; 7(6): e1002084. 
9 Sergouniotis PI, Robson AG, Li Z, Devery S, Holder GE, Moore AT et al. A phenotypic study of congenital stationary night blindness (CSNB) associated with mutations in the GRM6 gene. Acta Ophthalmol 2012; 90(3): e192-e197.

10 Jun G, Moncaster JA, Koutras C, Seshadri S, Buros J, McKee AC et al. delta-Catenin is genetically and bio logically associated with cortical cataract and future Alzheimer-related structural and functional brain changes. PLoS One 2012; 7(9): e43728.

11 Su S, Yao Y, Zhu R, Liang C, Jiang S, Hu N et al. The associations between single nucleotide polymorphisms of DNA repair genes, DNA damage, and age-related cataract: Jiangsu Eye Study. Invest Ophthalmol Vis Sci 2013; 54(2): 1201-1207.

12 Zhao J, Ellwein LB, Cui H, Ge J, Guan H, Lv J et al. Prevalence of vision impairment in older adults in rural China: the China Nine-Province Survey. Ophthalmology 2010; 117(3): 409-416.

13 Xu L, Li J, Cui T, Hu A, Fan G, Zhang R et al. Refractive error in urban and rural adult Chinese in Beijing. Ophthalmology 2005; 112(10): 1676-1683.

14 Hsi E, Chen KC, Chang WS, Yu ML, Liang CL, Juo SH et al. A functional polymorphism at the FGF10 gene is associated with extreme myopia. Invest Ophthalmol Vis Sci 2013; 54(5): 3265-3271.

15 Rada JA, Shelton S, Norton TT. The sclera and myopia. Exp Eye Res 2006; 82(2): 185-200.

16 Xu X, Li S, Xiao X, Wang P, Guo X, Zhang Q et al. Sequence variations of GRM6 in patients with high myopia. Mol Vis 2009; 15: 2094-2100.

17 Dryja TP, McGee TL, Berson EL, Fishman GA, Sandberg MA, Alexander KR et al. Night blindness and abnormal cone electroretinogram $\mathrm{ON}$ responses in patients with mutations in the GRM6 gene encoding mGluR6. Proc Natl Acad Sci USA 2005; 102(13): 4884-4889.

18 Xiao X, Jia X, Guo X, Li S, Yang Z, Zhang Q et al. CSNB1 in Chinese families associated with novel mutations in NYX. J Hum Genet 2006; 51(7): 634-640.

19 Koike C, Obara T, Uriu Y, Numata T, Sanuki R, Miyata K et al. TRPM1 is a component of the retinal ON bipolar cell transduction channel in the mGluR6 cascade. Proc Natl Acad Sci USA 2010; 107(1): 332-337.

20 Masu M, Iwakabe H, Tagawa Y, Miyoshi T, Yamashita M, Fukuda $\mathrm{Y}$ et al. Specific deficit of the ON response in visual transmission by targeted disruption of the mGluR6 gene. Cell 1995; 80(5): 757-765.

21 Tran-Viet KN, St Germain E, Soler V, Powell C, Lim SH, Klemm T et al. Study of a US cohort supports the role of ZNF644 and high-grade myopia susceptibility. Mol Vis 2012; 18: 937-944.

22 Lam CY, Tam PO, Fan DS, Fan BJ, Wang DY, Lee CW et al. A genome-wide scan maps a novel high myopia locus to 5p15. Invest Ophthalmol Vis Sci 2008; 49(9): 3768-3778.

23 Li YJ, Goh L, Khor CC, Fan Q, Yu M, Han S et al. Genomewide association studies reveal genetic variants in CTNND2 for high myopia in Singapore Chinese. Ophthalmology 2011; 118(2): 368-375.

24 Yu Z, Zhou J, Chen X, Zhou X, Sun X, Chu R. Polymorphisms in the CTNND2 gene and 11q24.1 genomic region are associated with pathological myopia in a Chinese population. Ophthalmologica 2012; 228(2): 123-129. 